\title{
Adding cognitive behavioural treatment to either low-carbohydrate or low-fat diets: differential short-term effects
}

\author{
Heriberto Rodriguez-Hernandez ${ }^{1,2}$, Uziel A. Morales-Amaya ${ }^{3}$, Ramon Rosales-Valdéz $z^{3}$, \\ Felipe Rivera-Hinojosa ${ }^{4}$, Martha Rodriguez-Moran ${ }^{1,2}$ and Fernando Guerrero-Romero ${ }^{1,2 *}$ \\ ${ }^{1}$ Biomedical Research Unit, Mexican Social Security Institute, Predio Canoas No. 100, Col. Los Angeles, 34067 Durango, \\ Dgo, Mexico \\ ${ }^{2}$ Research Group on Diabetes and Chronic Illnesses, D.A. Siqueiros 225 esq/Castañeda, Zona centro, 34000 Durango, \\ Dgo, Mexico \\ ${ }^{3}$ Psychology School, Jose Vasconcelos University, Calle Guadalupe No. 311, 34000 Durango, Dgo, Mexico \\ ${ }^{4}$ Nutrition Department, General Hospital of Mexican Social Security Institute at Durango, Predio Canoas y Ave. La Normal, \\ Col. Silvestre Dorador, 34067 Durango, Dgo, Mexico \\ (Received 1 December 2008 - Revised 13 May 2009 - Accepted 26 May 2009 - First published online 14 August 2009)
}

\begin{abstract}
To evaluate the efficacy of adding cognitive behavioural treatment (CBT) to either a low-carbohydrate (LC) diet or a low-fat (LF) diet in the treatment of weight loss of obese women, a randomised clinical intervention study was performed. A total of 105 healthy non-pregnant obese women (average age and BMI of 45.4 (SD 10.4) years and 36 (SD 4.3$) \mathrm{kg} / \mathrm{m}^{2}$ ) were randomly allocated to the CBT or control (C) groups; within each group, women were randomly selected to receive either the LC or LF diet during 6 months. The pre-planned primary trial endpoint was the weight loss. Differences between the groups were assessed using one-way ANOVA. There were three women ( $2 \cdot 8 \%)$ who dropped out, all of them in the CBT group. No differences in the anthropometric and laboratory characteristics at baseline were noted between women in the CBT ( $n$ 52) and control groups ( $n$ 50). Intention-to-treat analysis showed that weight loss in the CBT-LC (90 (SD $12 \cdot 3)$ to $82 \cdot 1$ (SD $12 \cdot 1) \mathrm{kg}$ ) and C-LC (89.4 (SD 10.0) to 85.8 (SD 9.8) kg) groups reached 8.7 and $4.0 \%$, respectively $(P<0.0001$ ), and in the CBT-LF (87.9 (SD 11.4 ) to 79.4 $(\mathrm{SD} 11.8) \mathrm{kg}$ ) and C-LF (88.8 (SD 14.5) to 85.3 (SD 14.3) $\mathrm{kg}$ ) groups it was 9.7 and $3.9 \%$, respectively $(P<0.05$ ). Weight loss was higher in the CBT-LF group than in the CBT-LC groups $(P=0.049)$. The present results showed that adding CBT to either the LF or LC diet produced significantly greater short-term weight loss in obese women compared with diet alone. These finding support the efficacy of CBT in breaking previous dietary patterns and in developing healthier attitudes that reinforce a healthier lifestyle.
\end{abstract}

Obesity: Cognitive behavioural treatment: Low-carbohydrate diets: Low-fat diets

Obesity, a consequence of overeating and lack of exercise, is an important clinical and public health burden worldwide. In $2005,23 \cdot 2 \%$ of the world's adult population was overweight, $9.8 \%$ was obese, and the estimated total numbers of overweight and obese adults were 937 million and 396 million, respectively. By 2030, if recent secular trends continue unabated, the estimated absolute numbers of overweight and obese individuals will be $2 \cdot 16$ billion and 1.12 billion $^{(1)}$. Because this increase in body weight is associated with a rise in CVD, type 2 diabetes, non-alcoholic fatty liver disease and hypertension ${ }^{(2,3)}$, programmes for the prevention and treatment of obesity should be a priority to face the challenge of this public health problem.

Both low-fat and low-carbohydrate diets have been shown to induce weight loss and reduce obesity-related co-morbidities; however, most but not all of the obese individuals who are participating in weight-loss strategies that involve diet and exercise reduce their initial body weight ${ }^{(4,5)}$, and the improvement in weight between groups is small ${ }^{(5)}$. Regarding diet, low-carbohydrate diets have been shown to promote a significantly greater weight loss than low-fat $\operatorname{diets}^{(6)}$, and lowcarbohydrate diets had more favourable overall outcomes on atherogenic dyslipidaemia and glycaemic control ${ }^{(7,8)}$. Although it is routinely indicated for individuals participating in programmes for weight loss, exercise per se induces a small weight reduction. The combination of both exercise and diet results in marginal benefits compared with diet alone ${ }^{(8,9)}$. Because the primary gauge for successful obesity treatment should be focused on health improvement instead of bodyweight reduction ${ }^{(10)}$, behavioural treatment emerges as a useful approach in helping obese individuals lose weight $^{(10,11)}$. Behavioural treatment aids in the identification

Abbreviations: BAI, Beck Anxiety Inventory; BDI, Beck Depression Inventory; CBT, cognitive behavioural treatment; CBT-LC, cognitive behavioural treatment with low-carbohydrate diet; CBT-LF, cognitive behavioural treatment with low-fat diet; C-LC, control group with low-carbohydrate diet; C-LF, control group with low-fat diet; LC, low-carbohydrate; LF, low-fat.

* Corresponding author: Dr Fernando Guerrero-Romero, fax +52 618 8132014, email guerrero_romero@hotmail.com 
of those factors that trigger overeating and the lack of physical activity, promotes the adoption of new responses, and reinforces the adoption of positive behaviours ${ }^{(12)}$. The goal of treatment is to initiate and maintain a process of problem solving to determine whether the removal or reduction of the causal factors promotes an appropriate manner of eating and greater weight loss ${ }^{(13)}$. The central principle in behaviour therapy is that patients must learn dietary restraint to resist unhealthy eating habits ${ }^{(13)}$. As a consequence, the behavioural approach is now integrated into most programmes for weight loss, inducing weight losses in the range of 9 to $13 \mathrm{~kg}^{(14,15)}$. However, studies indicate that $30-35 \%$ of patients regain weight in the first year after treatment and that 3-5 years later, at least $50 \%$ of participants have returned to their initial weight or more ${ }^{(16)}$.

Thus, among the strategies used to increase the rate of successful weight loss in adults, cognitive behavioural treatment (CBT), a triphasic focal psychotherapy intervention, is the most well-established psychotherapeutic treatment of problematic psychosocial functioning that characterises eating disorders and obesity ${ }^{(17)}$. In one of the studies using CBT strategies, Cresci et al. ${ }^{(18)}$ reported that the mean weight loss among subjects participating in a behavioural programme for the treatment of obesity was superior at 6 months compared with a similar group of subjects who received conventional treatment for obesity (2.0 (SD 3.9) v. $\left.0.8(\mathrm{SD} 2.5) \mathrm{kg} / \mathrm{m}^{2} ; P<0.05\right)$. Results showing the initial and long-term efficacy of CBT in the treatment of primary and secondary symptoms of binge-eating disorders ${ }^{(17,19)}$ and in the continuation of weight loss during follow-up ${ }^{(20-22)}$ are consistent.

However, to the best of our knowledge, there are no current data about what type of energy-reducing diet, when combined with CBT, produces the best initial treatment outcome. Thus, the aim of the present study was to evaluate the efficacy of adding CBT to either low-carbohydrate or low-fat diets in the treatment of weight loss of obese women.

\section{Experimental methods}

With the approval of the protocol by the Research Committee of the Mexican Social Security Institute, a randomised clinical intervention study was carried out in Durango City in northern Mexico, from July 2007 to June 2008.

\section{Participants}

Obese women from the same neighbourhood and similar social and economic strata were invited to participate in the study. A total of 105 obese women with an average age and BMI of 45.4 (SD 10.4) years (range 25-61 years) and $36(\mathrm{sD} 4 \cdot 3) \mathrm{kg} / \mathrm{m}^{2}$ (range $31 \cdot 2-47 \cdot 8 \mathrm{~kg} / \mathrm{m}^{2}$ ) were enrolled. All participants gave their written informed consent to participate in the study.

Pregnancy, hypothyroidism, heart failure, and renal and hepatic disease were exclusion criteria.

Obese women were assigned to study groups using twostage random procedures. In the first stage, a total of fiftyfive $(52.4 \%)$ women were randomly assigned to receive CBT, and fifty women were assigned to the control group. In the second stage, within each group, women were randomly selected to receive either low-carbohydrate (LC) or low-fat (LF) diets. Four groups were assigned for analysis: CBT-low-carbohydrate diet (CBT-LC); CBT-low-fat diet (CBT-LF); control group with low-carbohydrate diet (C-LC); control group with low-fat diet (C-LF).

The women in the study were followed for 6 months. The primary trial end-point was the percentage of weight loss. The final distribution of the population in the study is shown in Fig. 1.

\section{Treatment programme}

Because obesity is frequently associated with mood disorders, such as anxiety and depression ${ }^{(23)}$, all women in both groups received weekly psychological support for treatment or prevention of depression and anxiety. In addition, women in the CBT and control groups received weekly sessions of $1 \mathrm{~h}$ for diet and exercise advice. Additionally, women in the CBT group received an intervention that included behavioural strategies, cognitive skills and relapse prevention techniques to identify factors that trigger overeating and the lack of physical activity and for promoting the use of new responses to these triggers.

All sessions for women in both groups were individually conducted by trained personnel of the Biomedical Research Unit. Diaries of both diet and exercise were completed by participants and reviewed at each visit. Recommendations for exercise were walking, dancing, bicycling or swimming for $30 \mathrm{~min}$ per $\mathrm{d}$, on at least $5 \mathrm{~d}$ per week.

Total energy intake was calculated based on $125 \mathrm{~kJ} / \mathrm{kg}$ per d (30 kcal/kg per d) of ideal body weight. The LC diet was based on the following percentage of total energy intake per nutrient: $27 \%$ protein, $28 \%$ fat and $45 \%$ carbohydrate. The LF diet was based on $21 \%$ of daily energy intake from fat, less than $10 \%$ saturated fat, $25 \%$ protein and $54 \%$ carbohydrates $^{(24,25)}$. Adherence to diet and exercise was assessed every week by personal interviews with the female participant and a family member living with her.

Hepatic disease, serum creatinine level $\geq 15 \mathrm{mg} / \mathrm{l}$, severe life-limiting medical illness, active participation in other dietary programmes and the use of weight-loss drugs were exclusion criteria.

\section{Definitions}

Obesity was defined as a BMI $\geq 30 \mathrm{~kg} / \mathrm{m}^{2(26)}$. CBT, a triphasic focal psychotherapy, was based on behavioural strategies, learning cognitive skills and prevention techniques of relapse. CBT includes strategies for keeping food and activity records, activity of progressive relaxation, nutrition education, techniques for eating, strategies for recognising and solving problems and cognitive restructuring ${ }^{(27)}$.

Depression and anxiety were measured using the Beck Anxiety Inventory (BAI) and the Beck Depression Inventory (BDI) scales, which consist of twenty-one items each, that have been previously validated in the Mexican population. The BAI and BDI provide reliable information about characteristics, attitudes, and symptoms of anxiety and depression. Diagnosis of depression and anxiety was based on the following scale of BAI and BDI: 0-13 points; absent; $14-15$ points, mild; $16-19$ points, moderate; $\geq 20$ points, severe ${ }^{(28,29)}$. 


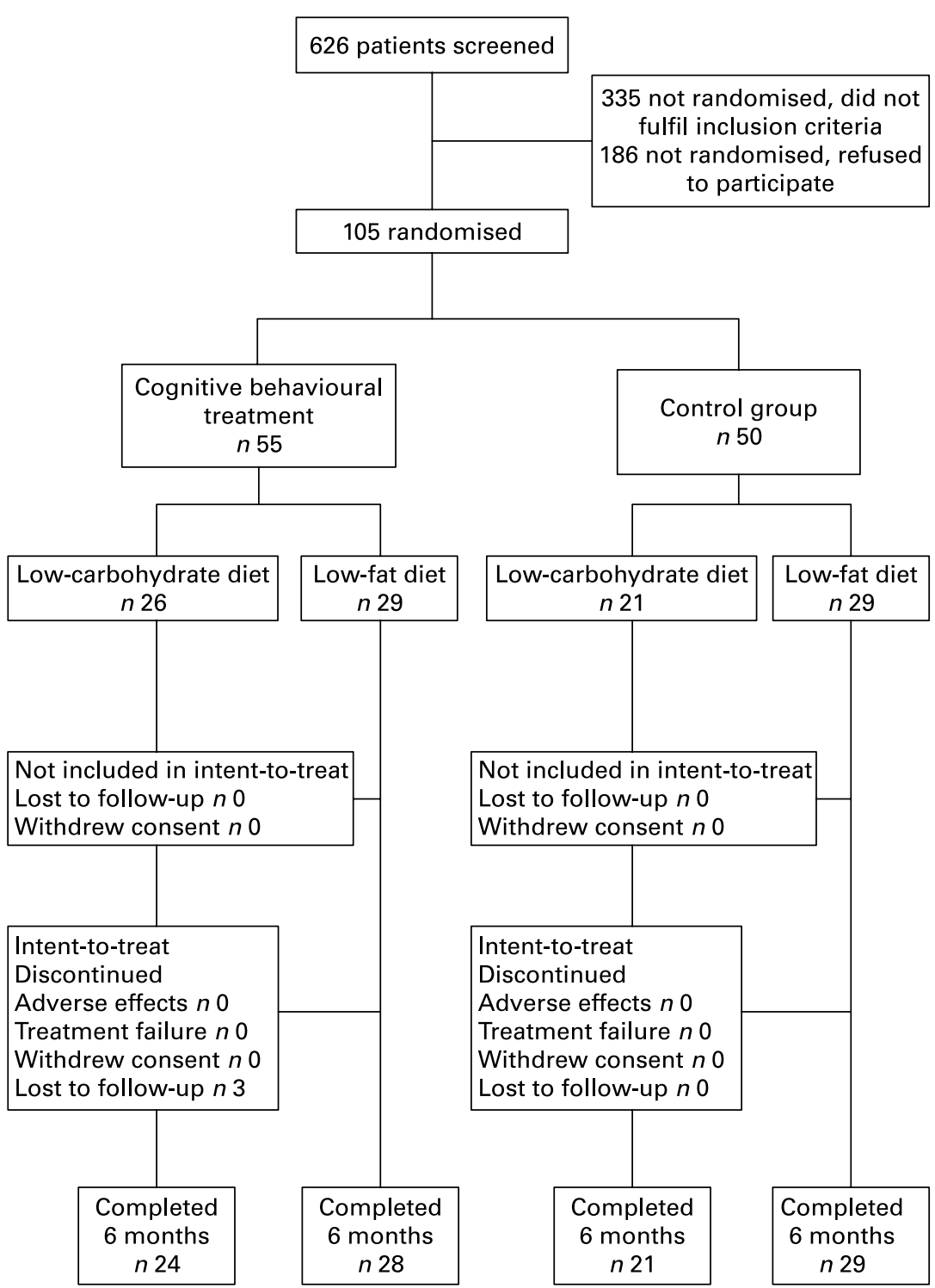

Fig. 1. Study participant flow diagram.

\section{Measurements}

The applied questionnaires covered demographic characteristics and medical conditions. Measurements included blood pressure, total body fat, waist circumference, weight and height.

In the standing position, weight and height were measured with the women in light clothing using a fixed scale with a stadiometer (Tanita TBF-215; Tanita, Tokyo, Japan). The precision of weight and height measurements was $0 \cdot 1 \mathrm{~kg}$ and $0.01 \mathrm{~m}$. BMI was calculated as weight $(\mathrm{kg})$ divided by height (m) squared. Waist circumference was measured halfway between the last rib and the iliac crest. Total body fat was measured by bioelectric impedance using a body composition analyser (Tanita TBF-215; Tanita) with $0.1 \%$ increments. Anthropometric measurements were performed at baseline and every month.

Using a Baumanometer (Microlife AG, Heerbrugg, Switzerland) and stethoscope (3M Littman Classic II; 3M Medica GmbH, Neuss, Germany), the technique used for the

measurement of blood pressure was the one that was recommended in the Seventh Report of the Joint National Committee on Prevention, Detection, Evaluation, and Treatment of High Blood Pressure ${ }^{(30)}$.

\section{Assays}

A venous whole-blood sample was collected during fasting. Serum glucose and TAG were determined by the glucoseoxidase and enzymic methods, respectively. Laboratory measurements were performed at baseline and post-test. The intra- and inter-assay variations were 2.1 and $1.5 \%$, respectively, for the glucose-oxidase method and 2.0 and $3.0 \%$, respectively, for the enzymic method. Measurements were performed in an Express 500 clinical chemistry autoanalyser (Ciba Corning, Diagnostic Corp., Overling, OH, USA). 
Table 1. Baseline characteristics of the women allocated to receive the low-carbohydrate (LC) or low-fat (LF) diet (Mean values and standard deviations)

\begin{tabular}{|c|c|c|c|c|c|c|c|c|c|c|c|c|c|c|}
\hline & \multicolumn{6}{|c|}{ LC } & \multicolumn{6}{|c|}{ LF } & \multirow[b]{3}{*}{$F$} & \multirow[b]{3}{*}{$P^{*}$} \\
\hline & \multicolumn{2}{|c|}{ All $(n 55)$} & \multicolumn{2}{|c|}{ CBT $(n 26)$} & \multicolumn{2}{|c|}{ Control (n 29) } & \multicolumn{2}{|c|}{ All $(n 50)$} & \multicolumn{2}{|c|}{ CBT $(n 21)$} & \multicolumn{2}{|c|}{ Control (n 29) } & & \\
\hline & Mean & SD & Mean & SD & Mean & SD & Mean & SD & Mean & SD & Mean & SD & & \\
\hline Age (years) & $45 \cdot 3$ & 9.9 & - & - & - & - & $45 \cdot 4$ & $10 \cdot 9$ & - & - & - & - & 1.61 & 0.207 \\
\hline Waist circumference $(\mathrm{cm})$ & 104.5 & $11 \cdot 0$ & 104.4 & $12 \cdot 3$ & 113 & 13.8 & $104 \cdot 6$ & $11 \cdot 7$ & $104 \cdot 3$ & 11.7 & 104.4 & $14 \cdot 8$ & 1.210 & 0.291 \\
\hline Weight (kg) & $89 \cdot 7$ & $11 \cdot 2$ & 90 & $12 \cdot 3$ & 89.4 & $10 \cdot 0$ & 88.3 & $13 \cdot 0$ & 87.9 & 11.4 & $88 \cdot 8$ & 14.5 & 0.005 & 0.945 \\
\hline BMI $\left(\mathrm{kg} / \mathrm{m}^{2}\right)$ & $36 \cdot 4$ & $4 \cdot 2$ & $36 \cdot 5$ & 4.3 & $36 \cdot 3$ & $4 \cdot 2$ & $35 \cdot 8$ & 4.4 & 35.5 & 3.5 & $36 \cdot 0$ & $5 \cdot 3$ & 0.014 & 0.905 \\
\hline Total body fat (\%) & 44.5 & 4.5 & 44.7 & 3.7 & $42 \cdot 2$ & $11 \cdot 2$ & 44.6 & 4.4 & 44.4 & $4 \cdot 1$ & $43 \cdot 8$ & $8 \cdot 8$ & 0.009 & 0.925 \\
\hline Fasting glucose $(\mathrm{mg} / \mathrm{l})$ & 957 & 165 & 980 & 164 & 930 & 167 & 1099 & 428 & 1029 & 416 & 1169 & 436 & 0.77 & 0.381 \\
\hline TAG (mg/l) & 1848 & 1134 & 1844 & 127 & 1852 & 989 & 1894 & 1219 & 1498 & 672 & 2276 & 1493 & 3.48 & 0.06 \\
\hline
\end{tabular}

$\mathrm{CBT}$, cognitive behavioural treatment.

${ }^{*} P$ value estimated by one-way ANOVA with Bonferroni post hoc test.

\section{Statistical analysis}

The pre-planned intention-to-treat analysis of the primary study end-point was done for all the randomly allocated participants (Fig. 1). Differences between the groups were assessed using the unpaired Student's $t$ test (Mann-Whitney $U$ test) and the $\chi^{2}$ test (for qualitative variables). Data between baseline and post-test within the same group were analysed using the paired Student's $t$ test (Wilcoxon test). Differences between more than two groups were analysed by one-way ANOVA with the Bonferroni post hoc $t$ test.

All data were processed and analysed using the statistical package SPSS for Windows version 15.0 (SPSS, Inc., Chicago, IL, USA).

\section{Results}

There were three women $(2 \cdot 8 \%)$ who dropped out, all from the CBT group (Fig. 1). Women in both groups received an average of 6795 (SD 12.64) kJ/d during the 6 months of treatment. Adherence to diet and exercise was reached by $91 \%$ of the women in both groups.

Women in the LF diet groups exhibited higher rates of diabetes $(17.5$ v. $2.0 \% ; P=0.009)$ and hypertension $(46.6 v$. $21.0 \% ; P=0.006)$ compared with women in the LC groups.
The baseline characteristics of participants are shown in Table 1. There were no significant differences between the groups for obesity measurements, fasting glucose, or TAG levels.

Of the study participants, twenty-six $(47 \cdot 3 \%)$ and twentynine $(52.7 \%)$ women were assigned to the CBT-LC and CBT-LF groups, respectively, and these study groups were compared with twenty-one $(42 \%)$ and twenty-nine $(58 \%)$ women in the C-LC and C-LF diet groups, respectively.

\section{Low-carbohydrate groups}

At baseline, there were no significant differences in body weight, BMI or body fat between the women in the CBTLC and C-LC groups. At post-test, women in the CBT-LC diet group significantly decreased their TAG concentrations compared with women in the C-LC group (Table 2).

At baseline, the rates of depression (54 and $62 \% ; P=0.82$ ) and anxiety (62 and $66 \% ; P=0.98)$ were similar in both the CBT-LC and C-LC groups; although the decrease in depression and anxiety between baseline and post-test was higher in the CBT-LC group, there were no significant differences between the groups $(-53.8$ and $-38.9 \%, P=0.48$ for depression; -60 and $-36.8 \%, P=0.29$ for anxiety in the CBT-LC and C-LC groups, respectively).

Table 2. Characteristics of the women who received the cognitive behavioural treatment (CBT) with the low-carbohydrate (LC) diet (CBT-LC) and of the control group with the LC diet (C-LC)

(Mean values and standard deviations)

\begin{tabular}{|c|c|c|c|c|c|c|c|c|c|c|}
\hline & \multicolumn{4}{|c|}{ CBT-LC } & \multicolumn{4}{|c|}{ C-LC } & \multirow[b]{3}{*}{$F$} & \multirow[b]{3}{*}{$P^{*}$} \\
\hline & \multicolumn{2}{|c|}{ Baseline } & \multicolumn{2}{|c|}{ Post-test§ } & \multicolumn{2}{|c|}{ Baseline } & \multicolumn{2}{|c|}{ Post-test§ } & & \\
\hline & Mean & SD & Mean & SD & Mean & SD & Mean & SD & & \\
\hline Waist circumference $(\mathrm{cm})$ & $104 \cdot 4$ & $12 \cdot 3$ & 98.7† & $10 \cdot 8$ & 113 & $13 \cdot 8$ & $108 \cdot 8$ & $11 \cdot 8$ & $2 \cdot 827$ & 0.04 \\
\hline Weight $(\mathrm{kg})$ & $90 \cdot 0$ & $12 \cdot 3$ & $82 \cdot 1 \dagger \dagger \dagger$ & $12 \cdot 1$ & 89.4 & $10 \cdot 0$ & $85.8 \dagger$ & $9 \cdot 8$ & $2 \cdot 450$ & 0.07 \\
\hline BMI $\left(\mathrm{kg} / \mathrm{m}^{2}\right)$ & $36 \cdot 5$ & $4 \cdot 3$ & 33.3†† & $4 \cdot 3$ & $36 \cdot 3$ & $4 \cdot 2$ & 34.9††† & $4 \cdot 0$ & 2.934 & 0.04 \\
\hline Total body fat (\%) & $44 \cdot 7$ & $3 \cdot 7$ & $43 \cdot 3$ & $4 \cdot 9$ & $42 \cdot 2$ & $11 \cdot 2$ & 41.7††† & $9 \cdot 4$ & 0.800 & 0.497 \\
\hline Fasting glucose (mg/l) & 980 & 164 & 953 & 235 & 930 & 167 & 927 & 172 & 0.393 & 0.758 \\
\hline TAG $(\mathrm{mg} / \mathrm{l})$ & 1844 & 127 & $1231 \dagger$ & 431 & 1852 & 989 & $1423 \ddagger$ & 639 & $2 \cdot 129$ & 0.109 \\
\hline
\end{tabular}

* $P$ value estimated by one-way ANOVA with Bonferroni post hoc test.

Mean value, within each group, was significantly different from that at baseline: $\dagger P<0.05$, $\dagger \dagger \dagger P<0.0005$ (paired $t$ test).

$\ddagger$ Mean value was significantly different from that of the CBT-LC group at post-test $(P<0.05)$.

$\S$ At 6 months after initiation of the diet treatment. 
Table 3. Characteristics of the women who received the cognitive behavioural treatment (CBT) with the low-fat (LF) diet (CBT-LF) and of the control group with the LF diet (C-LF)

(Mean values and standard deviations)

\begin{tabular}{|c|c|c|c|c|c|c|c|c|c|c|}
\hline & \multicolumn{4}{|c|}{ CBT-LF } & \multicolumn{4}{|c|}{ C-LF } & \multirow[b]{3}{*}{$F$} & \multirow[b]{3}{*}{$P^{\star}$} \\
\hline & \multicolumn{2}{|c|}{ Baseline } & \multicolumn{2}{|c|}{ Post-test§ } & \multicolumn{2}{|c|}{ Baseline } & \multicolumn{2}{|c|}{ Post-test§ } & & \\
\hline & Mean & SD & Mean & SD & Mean & SD & Mean & SD & & \\
\hline Waist circumference $(\mathrm{cm})$ & $104 \cdot 3$ & 11.7 & $100 \cdot 4 \dagger$ & $8 \cdot 8$ & 104.4 & $14 \cdot 8$ & $102 \cdot 7$ & $10 \cdot 6$ & $2 \cdot 827$ & 0.04 \\
\hline Weight $(\mathrm{kg})$ & 87.9 & 11.4 & $79.4+\dagger \dagger$ & $11 \cdot 8$ & 88.8 & 14.5 & 85.3†††‡ & $14 \cdot 3$ & $2 \cdot 831$ & 0.04 \\
\hline $\operatorname{BMI}\left(\mathrm{kg} / \mathrm{m}^{2}\right)$ & 35.5 & 3.5 & 32.0††† & 3.7 & $36 \cdot 0$ & $5 \cdot 3$ & 34.6†††‡ & $5 \cdot 2$ & 4.256 & 0.007 \\
\hline Total body fat (\%) & 44.4 & $4 \cdot 1$ & $40.8 t$ & $5 \cdot 3$ & 43.8 & $8 \cdot 8$ & 409 & 7.4 & 3.229 & 0.02 \\
\hline Fasting glucose $(\mathrm{mg} / \mathrm{l})$ & 1029 & 416 & 1014 & 288 & 1169 & 436 & 1160 & 450 & 1.014 & 0.390 \\
\hline TAG (mg/l) & 1498 & 672 & 1343 & 562 & 2276 & 1493 & $1893+t+\neq$ & 868 & 4.189 & 0.008 \\
\hline
\end{tabular}

${ }^{*} P$ value estimated by one-way ANOVA with Bonferroni post hoc test.

Mean value, within each group, was significantly different from that at baseline: $† P<0.05$, $\uparrow \dagger \dagger P<0.0005$ (paired $t$ test).

$\ddagger$ Mean value was significantly different from that of the CBT-LF group at post-test $(P<0.05)$.

$\S$ At 6 months after initiation of the diet treatment.

\section{Low-fat diet groups}

No significant differences were documented in weight, BMI and body fat between the women in the CBT-LF and C-LF groups at baseline. However, at post-test, women in the CBT-LF group had significantly decreased body fat, weight, BMI and TAG concentrations compared with women in the C-LF group (Table 3).

Similar to the results seen for the LC groups, at baseline, the rates of depression $(52.4$ and $62 \% ; P=0.70)$ and anxiety (76.1 and $65.5 \% ; P=0.62$ ) were similar in the CBT-LF and C-LF groups; however, at post-test, the decrease of depression and anxiety, although higher in the CBT-LF group, showed no significant differences $(-63.6$ and $-44.4 \%, P=0.44$ for depression; -56.2 and $-36.8 \%, P=0.32$ for anxiety in the CBT-LF and C-LF groups, respectively).

Figure 2 shows the proportion of weight loss between baseline and post-test in the groups in the study. Weight loss among women in the CBT-LC and C-LC diet groups was 8.7 and $4.0 \%$, respectively $(P<0.0001)$, whereas in the CBT-LF and C-LF diet groups, weight loss reached 9.7 and $3.9 \%$, respectively $(P<0.05)$. The weight loss was greater among women in the CBT-LF group compared with women in the CBT-LC group $(P=0.049)$; on the other hand, the difference between the C-LC and C-LF groups was not significant $(P=0.75)$.

\section{Discussion}

The present results showed that adding CBT to either an energy-restricted, low-carbohydrate diet or to an energyrestricted, low-fat diet produces significantly greater shortterm weight loss compared with diet alone. In addition, the present results also show that adding CBT to a low-fat diet produces higher short-term weight loss than adding CBT to a low-carbohydrate diet.

Because obesity has emerged as a major public health problem, several recommendations for diet and lifestyle changes for the prevention of obesity and its complications have been made ${ }^{(31)}$. As one of the multiple strategies for weight loss, a behavioural programme could facilitate compliance in the adoption of required lifestyle changes ${ }^{(32)}$, identify those factors that trigger inappropriate eating and unsuitable habits of physical activity, and promote learning for achieving realistic goals for lifestyle changes ${ }^{(33)}$.

Individuals who combine energy-restriction diets and exercise with CBT may expect to lose about 5 to $10 \%$ of pre-intervention body weight over a period of 4 to 6 months ${ }^{(17,34)}$. Foster et al. ${ }^{(11)}$ reviewed several weight-loss studies based on behavioural strategies showing an average weight loss of $9.6 \mathrm{~kg}$ during the first 21 weeks of treatment. In accordance with previous reports, the present study provides evidence that weight loss is greater among obese
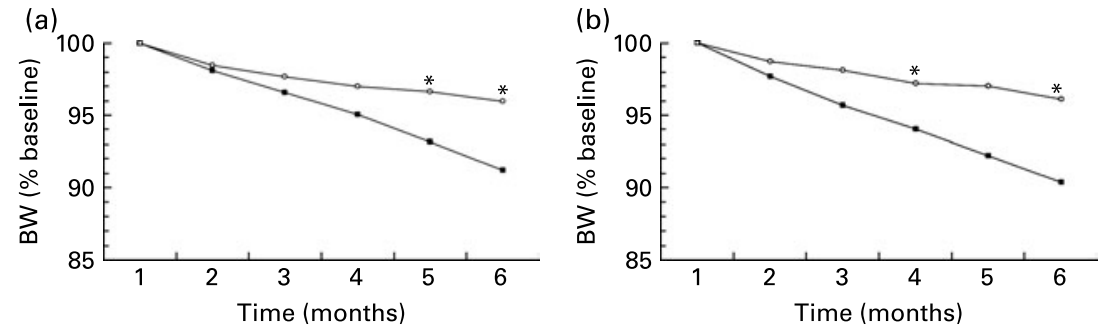

Fig. 2. Body-weight (BW; \% of baseline) in women with $(\mathbf{\square})$ and without $(O)$ cognitive behavioural treatment who received a low-carbohydrate diet (a) or a low-fat diet (b). At 6 months of treatment, weight loss in the women who received cognitive behavioural treatment and the low-fat diet was significantly lower than weight loss of the women in the cognitive behavioural treatment who received the low-carbohydrate diet. On the other hand, there were no significant differences between the women who received the low-fat and the low-carbohydrate diet, in the control groups. ${ }^{*}$ Mean value was significantly different from that of the women who received the cognitive behavioural treatment $(P<0.05)$. 
individuals undergoing CBT and that an important weight loss takes place in the first months of treatment. Furthermore, to the best of our knowledge, the present study is the first that includes a CBT component in a unique design that allowed us to evaluate the efficacy of adding CBT to different types of diets; our finding suggests that adding CBT to the management of obesity improves outcomes across different diets.

An increased focus on the emotional and cognitive processes associated with overeating has led to the detection of patterns that are potential causal factors of obesity. In this regard, the goal of $\mathrm{CBT}$ is to initiate and maintain a process of problem solving to determine whether the removal or reduction of the putative causal factors results in more prudent weight-management behaviours ${ }^{(13)}$. After achieving a desirable weight, patients are encouraged to maintain body weight through a programme for continuing support. The present study shows that, irrespective of the type of diet, CBT strategies improve the outcomes of programmes for weight loss, a finding that supports the potential synergies of CBT and energy-restricted diets ${ }^{(35-37)}$. Therefore, the combination of therapeutic strategies that improve a patient's knowledge of skills to avoid behaviours that lead to excessive intake with the low-energy diets is a promising approach for the treatment of obesity that is feasible in daily routine primary care.

In addition, women who received CBT support significantly reduced their serum TAG levels compared with the correspondent control groups. Previously, it has been shown that both low-fat and short-term low-carbohydrate weight-loss diets significantly decreased postprandial lipaemia and resulted in similar changes in fasting TAG levels. In this way, a decrease in body mass has been suggested to be the main factor involved in the improved postprandial lipaemia, independent of diet composition ${ }^{(38)}$. In this regard, the greater reduction in the TAG levels that was observed among women who received CBT support in the present study could be related to the greater weight loss and to the positive changes in cognitive skills that CBT promotes for developing healthier attitudes that reinforce a healthier lifestyle. Other studies highlight that obese subjects with diabetes or the metabolic syndrome show a relative improvement in TAG levels on a carbohydrate-restricted diet than on a fat-restricted $\operatorname{diet}^{(6)}$.

Controversy about the optimal type of diet for weight loss is not related solely to metabolic outcome. In this regard, some studies show that a low-carbohydrate diet promotes higher weight loss than low-fat diets ${ }^{(6,39)}$, but others highlight that a fat-restricted diet provides a high degree of satiety and promotes a higher reduction of body weight and better longterm adherence ${ }^{(40)}$. Further research is needed to understand why diets with similar energy restriction but different macronutrient compositions produce different effects on weight loss.

Limitations of the present study that deserve to be mentioned are the following: (a) since we only studied women, the present results cannot be applied to men; (b) the short period of intervention prevented the evaluation of the long-term efficacy of combining CBT and a low-fat diet for weight loss; although similar results have been reported in other populations that show that the maximum weight loss is usually achieved during the initial phases of treatment, the long-term clinical efficacy of CBT as a strategy for weight loss remains to be established.

\section{Conclusion}

The present results showed that adding CBT to either a low-fat diet or a low-carbohydrate diet produced significantly greater short-term weight loss in obese women compared with diet alone. These findings support the efficacy of CBT in breaking previous dietary patterns and in developing healthier attitudes that reinforce a healthier lifestyle.

\section{Acknowledgements}

The present study was supported by grants from the Fundación IMSS, A.C.

H. R.-H. contributed with the original idea and design of the study; U. A. M.-A. and R. R.-V. designed and applied the treatment for depression and anxiety; F. R.-H. contributed with the design of diets used; M. R.-M. performed the statistical analysis; F. G.-R. contributed with the statistical analysis and wrote the manuscript draft. All the authors approved the final manuscript draft.

None of the authors has any conflict of interest.

\section{References}

1. Kelly T, Yang W, Chen CS, et al. (2008) Global burden of obesity in 2005 and projections to 2030. Int J Obes (Lond) 32, $1431-1437$.

2. Ogden CL, Yanovski SZ, Carroll MD, et al. (2007) The epidemiology of obesity. Gastroenterology 132, 2087-2102.

3. Ogden CL, Carroll MD, Curtin LR, et al. (2006) Prevalence of overweight and obesity in the United States, 1999-2004. JAMA 295, 1549-1555.

4. Klein S (2004) Clinical trial experience with fat-restricted vs carbohydrate-restricted weight-loss diets. Obes Res 12, $141 S-144 S$.

5. Norris SL, Zhang X, Avenell A, et al. (2004) Long-term effectiveness of lifestyle and behavioral weight loss interventions in adults with type 2 diabetes: a meta-analysis. Am J Med 117, 762-774.

6. Samaha FF, Iqbal N, Seshadri P, et al. (2003) A lowcarbohydrate as compared with low-fat diet in severe obesity. $N$ Engl J Med 348, 2074-2081.

7. Stern L, Iqbal N, Seshadri P, et al. (2004) The effects of lowcarbohydrate versus conventional weight loss diet in severely obese adults: one-year follow-up of a randomized trial. Ann Intern Med 140, 778-785.

8. Eckel RH (2008) Nonsurgical management of obesity in adults. $N$ Engl J Med 358, 1941-1950.

9. Nordmann AJ, Nordmann A, Briel M, et al. (2006) Effects of low-carbohydrate vs low-fat diets on weight loss and cardiovascular risk factors: a meta-analysis of randomized controlled trials. Arch Intern Med 166, 285-293.

10. Kazaks A \& Stern JS (2003) Obesity treatments and controversies. Diabetes Spectr 16, 231-235.

11. Foster GD, Makris AP \& Bailer BA (2005) Behavioral treatment of obesity. Am J Clin Nutr 82, 230S-235S.

12. Simon GE, Von Korff M, Saunders K, et al. (2006) Association between obesity and psychiatric disorders in the US adult population. Arch Gen Psychiatry 63, 824-830.

13. Foreyt JP \& Goodrick GK (1993) Evidence for success of behavior modification in weight loss and control. Ann Intern Med 119, 698-701.

14. Brownell KD \& Kramer FM (1989) Behavioral management of obesity. Med Clin North Am 73, 185-201. 
15. Björvell H \& Rössner S (1985) Long term treatment of severe obesity: four year follow up of results of combined behavioural modification programme. $\mathrm{Br}$ Med J (Clin Res Ed) 291, 379-382.

16. Wadden T \& Foster GD (2000) Behavioral treatment of obesity. Med Clin North Am 84, 441-461.

17. Wilfley DE, Welch RR, Stein RI, et al. (2002) A randomized comparison of group cognitive-behavioral therapy and group interpersonal psychotherapy for the treatment of overweight individuals with binge-eating disorder. Arch Gen Psychiatry 59, 713-721.

18. Cresci B, Tesi F, La Ferlita T, et al. (2007) Group versus individual cognitive-behavioral treatment for obesity: results after 36 months. Eat Weight Disord 12, 147-153.

19. Munsch S, Biedert E, Meyer A, et al. (2007) A randomized comparison of cognitive behavioral therapy and behavioral weight loss treatment for overweight individuals with binge eating disorder. Int J Eat Disord 40, 102-113.

20. Van Dorsten B \& Lindley EM (2008) Cognitive and behavioral approaches in the treatment of obesity. Endocrinol Metab Clin North Am 37, 905-922.

21. Sbrocco T, Nedegaard RC, Stone JM, et al. (1999) Behavioral choice treatment promotes continuing weight loss: preliminary results of a cognitive-behavioral decision-based treatment for obesity. J Consult Clin Psychol 67, 260-266.

22. Golay A, Buclin S, Ybarra J, et al. (2004) New interdisciplinary cognitive-behavioural-nutritional approach to obesity treatment: a 5-year follow-up study. Eat Weight Disord 9, 29-34.

23. Onylike CH, Crum RM, Lee HB, et al. (2003) Is obesity associated with major depression? Result from the Third National Health and Nutrition Examination Survey. Am J Epidemiol 158, 1139-1147.

24. Lichtenstein AH, Appel LJ, Brands M, et al. (2006) Diet and lifestyle recommendations revision 2006: a scientific statement from the American Heart Association Nutrition Committee. Circulation 114, 82-96.

25. Yancy WS Jr, Olsen MK, Guyton JR, et al. (2004) A lowcarbohydrate, ketogenic diet versus a low-fat diet to treat obesity and hyperlipidemia. A randomized, controlled trial. Ann Intern Med 140, 769-777.

26. Bantle JP, Wylie-Rosett J, Albright AL, et al. (2006) Nutrition Recommendations and Interventions for Diabetes - 2006: a position statement of the American Diabetes Association. Diabetes Care 29, 2140-2157.
27. Wadden T \& Foster GD (2002) Behavioral treatment of obesity. Med Clin North Am 84, 441-461.

28. Beck AT, Emery G \& Greenberger R (1985) Anxiety Disorders and Phobias: a Cognitive Perspective. New York: Basic Books.

29. Beck AT, Rush A, Shaw B, et al. (1979) Cognitive Therapy of Depression. New York: Guilford Press.

30. Chobanian AV, Bakris GL, Black HR, et al. (2003) The Seventh Report of the Joint National Committee on Prevention. Detection, Evaluation, and Treatment of High Blood Pressure: the JNC 7 report. JAMA 289, 2560-2572.

31. Foster GD, Wyatt HR, Hill JO, et al. (2003) A randomized trial of a low carbohydrate diet for obesity. $N$ Engl J Med 348, 2082-2090.

32. Wadden TA, Butryn ML \& Wilson C (2007) Lifestyle modification for the management of obesity. Gastroenterology 132, 2226-2238

33. Foster GD (2006) Clinical implications for the treatment of obesity. Obesity 14, 182S-185S

34. Yanovski SZ \& Yanovski JA (2002) Obesity. N Engl J Med 346, 591-602.

35. Eldredge KL, Stewart Agras W, Arnow B, et al. (1997) The effects of extending cognitive-behavioral therapy for binge eating disorder among initial treatment nonresponders. Int $J$ Eat Disord 21, 347-352.

36. Nauta H, Hospers H, Kok G, et al. (2000) A comparison between a cognitive and a behavioral treatment for obese binge eaters and obese non-binge eaters. Behav Ther 31, 441-461.

37. Shelley-Ummenhofer J \& MacMillan PD (2007) Cognitivebehavioural treatment for women who binge eat. Can J Diet Pract Res 68, 139-142.

38. Volek JS, Sharman MJ, Gómez AL, et al. (2004) Comparison of a very low-carbohydrate and low-fat diet on fasting lipids, LDL subclasses, insulin resistance, and postprandial lipemic responses in overweight women. J Am Coll Nutr 23, $177-184$

39. Shai I, Schwarzfuchs D, Henkin Y, et al. (2008) Weight loss with low-carbohydrate. Mediterranean or low-fat diet. $N$ Engl J Med 359, 229-241.

40. Knopp RH, Walden CE, Retzlaff BM, et al. (1997) Long-term cholesterol-lowering effects of 4 fat-restricted diets in hypercholesterolemic and combined hyperlipidemic men. JAMA 278, $1509-1515$. 